\title{
Aspects of the circle composition operation in rings
}

\author{
HELEN L. Chick
}

In this thesis we investigate some of the properties of the circle composition (or adjoint) operation in rings, where the operation $\circ$ is defined by $a \circ b=a+b+a b$. In an arbitrary ring, $R$, the properties of addition and multiplication imply that $(R, \circ)$ is a semigroup. The Jacobson radical of a ring is the largest ideal in which the circle composition operation gives rise to a group. Quasiregular rings, in which the whole ring is the Jacobson radical, include the nil (and hence also nilpotent) rings.

If a quasiregular ring's multiplication is commutative then the circle composition operation commutes; the ring then has two Abelian group structures associated with it. The main purpose of this thesis is to investigate whether or not the two groups can be isomorphic and, if so, what implications this has for the ring. We use $\mathcal{K}$ to denote the class of all rings in which $(R, \circ)$ is isomorphic to $(R,+)$. That there are rings having isomorphic additive and circle composition groups is obvious: zero rings (where multiplication is trivial) clearly have the desired property, since the additive and circle composition groups coincide. There are also non-trivial examples and we illustrate the construction of some, including the so-called quasifields which are constructed on partially ordered sets. It might be suspected that for these less trivial examples the isomorphism between addition and circle composition will still force multiplication to behave in a nearly trivial way, so that perhaps such rings are nil or nilpotent. This need not be the case as there is a ring in $\mathcal{K}$ which has no zero divisors. In fact, we show that there exist rings in $\mathcal{K}$ which are nilpotent but not zero rings, nil but not nilpotent, and quasiregular without being nil.

We also construct a family of examples by taking finite Abelian groups and determining when they can form the additive group of a non-trivial ring in $\mathcal{K}$. If $p$ is a prime we can show that there is only the trivial $\mathcal{K}$-ring with $\mathbf{Z}_{p}$ as the additive group, we also show that $\mathbf{Z}_{\mathbf{4}}$ and $\mathbf{Z}_{2} \oplus \mathbf{Z}_{2}$ do not support non-trivial rings in $\mathcal{K}$. Of the remaining finite groups, for those of the form $\mathbf{Z}_{p^{n}}$ we show exactly how to obtain non-trivial examples of rings in $\mathcal{K}$ and indicate the number of non-isomorphic classes that arise for

Received 22nd August, 1997

Thesis submitted to the University of Tasmania, December 1996. Degree approved, March 1997. Supervisor: Dr. B.J. Gardner.

Copyright Clearance Centre, Inc. Serial-fee code: 0004-9729/98 \$A2.00+0.00. 
a given group; while for other finite Abelian groups we indicate how to obtain at least one non-trivial example of a $\mathcal{K}$-ring using direct sums or other constructions.

We examine the algebraic properties of the class $\mathcal{K}$, including the question of its inheritance under ring theoretic constructions. In particular, we show that $\mathcal{K}$ is not a radical class, that it is closed under direct products, but that it is not hereditary (closed under taking ideals) nor closed under homomorphisms nor taking quasiregular subrings. There are, however, certain subclasses of $\mathcal{K}$ which are better behaved, including, for example, rings which are algebras over $\mathbf{Z}_{p}$ or $\mathbf{Q}$ and the rings constructed on certain finite groups.

For commutative nilpotent rings we prove the existence of a polynomial hornomorphism between the additive and circle composition groups, which in certain circumstances will be an isomorphism. We show, too, that all finitely generated nilpotent $\mathbf{Q}$-algebras and $\mathbf{Z}$-algebras are in $\mathcal{K}$. The former result allows us to demonstrate that all commutative nil $\mathbf{Q}$-algebras are in $\mathcal{K}$.

We conclude by considering a family of ring examples in which the circle composition semigroup is regular, rather than a group. Our construction is developed from the idea behind the quasifield construction and also generalised power series rings. We investigate the existence of nilpotence in such rings, and show that, like $\mathcal{K}$, the class of rings in which $(R, 0)$ is a regular semigroup is not a radical class. This result also holds for the stronger property that $(R, 0)$ is a union of groups.

We note that many of the results have appeared in $[\mathbf{1}, \mathbf{2}, \mathbf{3}]$ and will appear in $[\mathbf{4}]$.

\section{REFERENCES}

[1] H.L. Chick, 'Quasi-division rings - Some examples of quasiregular rings', in Theory of Radicals (Proc. Conf. Szekszárd, 1991), (L. Márki and R. Wiegandt, Editors), Colloq. Math. Soc. J. Bolyai 61 (North-Holland, Amsterdam, 1993), pp. 35-59.

[2] H.L. Chick, 'Rings with isomorphic additive and circle composition groups', in Rings and radicals (Proceedings of the International Conference, Shijiazhuang 1994), (B.J. Gardner, Liu Shaoxue and R. Wiegandt, Editors), Pitman Research Notes in Mathematics 346 (Longman, 1996), pp. 160-169.

[3] H.L. Chick, 'The properties of some rings having isomorphic additive and circle composition groups', Austral. Math. Soc. Gaz. 23 (1996), 112-117.

[4] H.L. Chick and B.J. Gardner, 'Commutative quasiregular rings with isomorphic additive and circle composition groups, II: Rational algebras', Comm. Alg. (to appear).

Department of Mathematics

University of Tasmania

GPO Box 252-37

Hobart, Tas. 7001

Australia

e-mail: chick@hilbert.maths.utas.edu.au 